\title{
EGFR siRNA lipid nanocapsules efficiently transfect glioma cells in vitro
}

\author{
Pauline Resnier ${ }^{\mathrm{a}, \mathrm{b}}$, Stephanie David ${ }^{\mathrm{c}}$, Nolwenn Lautram ${ }^{\mathrm{a}, \mathrm{b}}$, Gaëtan J.-R. Delcroix ${ }^{\mathrm{d}, \mathrm{e}}$, \\ Anne Clavreul ${ }^{a, b, f}$, Jean-Pierre Benoit ${ }^{a, b}$, Catherine Passirani ${ }^{a, b, *}$ \\ a LUNAM - Université d'Angers, F-49933 Angers, France \\ b INSERM U1066 - Micro et Nanomédecines Biomimétiques, Angers, France \\ c EA 6592 - Nanomédicaments et nanosondes, Université François-Rabelais, Tours, France \\ d University of Miami Tissue Bank, Interdisciplinary Stem Cell Institute, Department of Othopaedics, University of Miami Miller School of Medecine, Miami, \\ FL, USA \\ e Geriatric Research, Education, and Clinical Center and Research Service, Bruce W.Carter Veterans Affairs Medical Center, Miami, FL, USA \\ ${ }^{\mathrm{f}}$ Département de Neurochirurgie, Centre Hospitalier Universitaire d'Angers, F-49100 Angers, France
}

\section{A R T I C L E I N F O}

\section{Article history:}

Received 18 February 2013

Received in revised form 22 March 2013

Accepted 2 April 2013

Available online $\mathrm{xxx}$

\section{Keywords:}

Nanomedicine

Brain tumor

Gene therapy

CH50

U87MG

\begin{abstract}
A B S T R A C T
Glioma are the most common malignant tumors of the central nervous system and remain associated with poor prognosis, despite the combination of chemotherapy and radiotherapy. EGFR targeting represents an interesting strategy to treat glioma. Indeed, a high level of endothelial growth factor receptors expression (EGFR), involved in the malignancy of the tumor, has been observed in glioma. Our strategy consisted in using EGFR siRNA entrapped into lipid nanocapsules (LNCs) via cationic liposomes. In vitro analyses on U87MG human glioma cells were performed to evaluate firstly the capacity of LNCs to efficiently deliver the siRNA and secondly the effect of EGFR siRNA targeting on U87MG proliferation. Then, the complement protein consumption was evaluated by $\mathrm{CH} 50$ assays to verify the suitability of the siRNA LNCs for systemic administration. The EGFR siRNA LNCs exhibited an adequate size lower than $150 \mathrm{~nm}$ as well as a neutral surface charge. The IC50 profile together with the $63 \%$ of protein extinction demonstrated the significant action of EGFR siRNA LNCs compared to scrambled LNCs. Dose and time-dependent survival assays showed a decrease of U87MG growth evaluated at 38\%. Finally, low complement consumption demonstrated the suitability of EGFR siRNA LNCs for intravenous injection. In conclusion, EGFR siRNA LNCs demonstrated their capacity to efficiently encapsulate and deliver siRNA into U87MG human glioma cells, and will therefore be usable in the future for in vivo evaluation.
\end{abstract}

(c) 2013 Elsevier B.V. All rights reserved.

\section{Introduction}

Malignant brain tumors are often associated with poor prognosis. The low survival median, of only 15 months in the case of glioblastoma (GBM), despite the use of surgery, chemotherapy with temozolomide (Temodal ${ }^{\circledR}$ ) and radiotherapy, has encouraged research for new treatment (Stupp and Roila, 2009). GBM are the most aggressive form of malignant brain tumors, and are characterized by a high infiltration capacity in healthy brain tissue leading to difficult tumor resection. The endothelial growth factor receptor (EGFR) has been described as an oncogene in many types of cancer, including GBM (Yang et al., 2012). The over-expression and/or amplification of EGFR protein, described in half-primary GBM cases, has been associated with abnormal

\footnotetext{
* Corresponding author at: INSERM U1066, IBS-CHU, 4 rue Larrey, 49933 Angers Cedex 9, France. Tel.: +33 244688 534; fax: +33 244688546.

E-mail address: catherine.passirani@univ-angers.fr (C. Passirani).
}

proliferation and a chemo/radioresistance phenomenon (Hatanpaa et al., 2010; Watanabe et al., 1996). To combat this drug resistance, various inhibitors of EGFR, such as antibodies (Cetuximab ${ }^{\circledR}$ ) and more recently tyrosine kinase inhibitors (TKI) $\left(\right.$ Gefitinib $\left.^{\circledR}\right)$, have been developed and tested in numerous in vitro and in vivo models. These studies have proved the relevance of EGFR targeting in GBM with anti-proliferative properties. Moreover, its impact on glioma stem cells was also demonstrated recently (Geoerger et al., 2008; Jin et al., 2013). However, despite numerous clinical trials, none of these inhibitors have been approved yet for clinical glioma treatment (Joshi et al., 2012; Taylor et al., 2012; Thiessen et al., 2010). The low efficiency of these candidate drugs could be explained by the specific biological environment in GBM. In fact, a resistance mechanism against antibodies or TKI usually appears in the form of variant protein expression, rendering the therapeutic approach ineffective. To prevent this phenomenon, the RNAi mechanism could represent a new opportunity thanks to the use of specific siRNA tools that do not depend on protein interaction, 3D conformation, or protein variants (Fire et al., 1998). Indeed, the siRNA used in this study was 
described as naturally acting through the micro RNA pathway to inhibit proteins like EGFR (Michiue et al., 2009).

Systemic delivery to the brain remains a challenge due to the presence of the blood brain barrier (BBB) that strongly limits the exchanges between blood and neuronal cells. Chemical or therapeutic agents (such as TKI, antibodies, and siRNA) cannot cross, or only partially, this membrane by passive transport (Wang et al., 2012). For this reason, the development of synthetic carriers represents a great opportunity to ameliorate the passage through the $\mathrm{BBB}$, especially for hydrophilic drugs. Thanks to specific targeting, nanomedicines could improve biodistribution into the brain after systemic injection by (i) the passive strategy with enhanced permeability and retention (EPR) effect (Maeda, 2001); (ii) by the active strategy with ligand grafting on the surface of the delivery system, such as transferrin (Beduneau et al., 2008).

Considering the systemic nucleic acid transport, nanocarriers are necessary to protect them from the blood compartment. In fact, nucleases and the immune system can rapidly degrade siRNA. The delivery systems can enhance the half-life of siRNA into blood and favor their accumulation into targeted organs (Morille et al., 2010). Numerous nanomedicines have been developed to encapsulate siRNA, such as liposomes, micelles, and dendrimers. The majority of these systems were made of a mixture of cationic polymers and lipid components entrapping siRNA thanks to electrostatic interactions. Lipid nanocapsules (LNCs) consisting of a lipid liquid core of triglycerides and a rigid shell of lecithin and polyethylene glycol, have been developed in our laboratory (Heurtault et al., 2002). The simple formulation process is based on phase-inversion of an emulsion. These LNCs have recently been modified to encapsulate DNA, complexed with cationic lipids forming lipoplexes, and have shown efficient in vitro and in vivo effects in different animal models (David et al., 2012a,b; Morille et al., 2011). In our recent work, these delivery systems were adapted to siRNA encapsulation (David et al., 2012c). We tested these siRNA LNCs on glioma cells in order to prove the efficient delivery of siRNA into the cytoplasm and the effect of EGFR inhibition by siRNA on U87MG human glioma cells.

\section{Materials and methods}

\subsection{SiRNA LNC formulation}

Lipid nanocapsules (LNCs) were formulated, as described before (Heurtault et al., 2002), by mixing 20\%, w/w Labrafac WL 1349 (caprylic-capric acid triglycerides, Gatefossé S.A. Saint-Priest, France), 1.5\%, w/w Lipoid S75-3 (Lipoid GmbH, Ludwigshafen, Germany), 17\%, w/w Kolliphor ${ }^{\circledR}$ HS 15 (BASF, Ludwigshafen, Germany), 1.8\%, w/w NaCl (Prolabo, Fontenay-sous-Bois, France) and $59.8 \%, w / w$ water (obtained from a Milli-Q system, Millipore, Paris, France) together under magnetic stirring. Briefly, three temperature cycles between 60 and $95^{\circ} \mathrm{C}$ were performed to obtain phase inversions of the emulsion. A subsequent rapid cooling and dilution with ice cooled water $(1: 1.4)$ at the phase-inversion temperature (PIT) led to blank LNC formation.

To obtain siRNA LNCs, the water in the last step was replaced by lipoplexes, i.e. SiRNA as EGFR siRNA (sense sequence: 5'-CACAGUGGAGCGAAUUCCU-3'; Eurogentec, Seraing, Belgium) and control (scrambled) siRNA (sense sequence: 5'-UCUACGAGGCACGAGACUU-3'; Eurogentec, Seraing, Belgium) complexed with cationic liposomes in a defined charge ratio of cationic lipid charge versus anionic siRNA charge ( \pm ratio). During this work, liposome LNCs, i.e. LNCs with liposome introduction at the last step without siRNA, were also used as control vehicle.

For liposome preparation, a cationic lipid DOTAP (1.2-dioleyl-3trimethylammoniumpropane) (Avanti ${ }^{\circledR}$ Polar Lipids Inc., Alabaster, AL, USA), solubilized in chloroform, was weighted in the ratio
1/1 (M/M) with the neutral lipid DOPE (1.2-dioleyl-sn-glycero-3phosphoethanolamine) (Avanti ${ }^{\circledR}$ Polar Lipids Inc., Alabaster, AL, USA) to obtain a final concentration of $30 \mathrm{mM}$ of cationic lipid charge, considering the number of lipid charges per molecule ( 1 for DOTAP). After chloroform evaporation under vacuum, deionized water was added to hydrate the lipid film over night at $4{ }^{\circ} \mathrm{C}$ which was sonicated the day after.

\subsection{Characterization}

The size and Zeta potential of LNCs were measured by the Dynamic Light Scattering (DLS) method using a Malvern Zetasizer ${ }^{\circledR}$ apparatus (Nano Series ZS, Malvern Instruments S.A., Worcestershire, UK) at $25^{\circ} \mathrm{C}$, in triplicate, after dilution in a ratio of $1: 200$ with deionized water.

Moreover, a spectrophotometric method, based on work recently described by David et al. (2012c), was used to evaluate the encapsulation efficiency. Briefly, siRNA LNCs were mixed with chloroform and water to separate respectively hydrophilic and lipophilic components, sodium hydroxide to destabilize lipoplexes and absolute ethanol to destroy LNCs. After multiple centrifugations, four compartments were obtained: free siRNA, free lipoplexes (i.e. siRNA associated with liposomes), encapsulated siRNA and encapsulated lipoplexes into LNCs. To determine the concentration of siRNA, the optical density (Morille et al.) of each sample were determined at $260 \mathrm{~nm}$ by UV spectrophotometer (UV2600, Shimadzu, Noisiel, France) in triplicate conditions. These physico-chemical parameters (size, charge surface and encapsulation) were characterized after formulation and 1 month later to study their stability.

\subsection{Cell culture}

Human glioma cell line U87MG and human macrophage/ monocyte THP-1 were obtained from ATCC. U87MG cells were grown in Minimum Essential Medium Eagle (Lonza, Verviers, Belgium) supplemented with $10 \%$ of fetal bovine serum (Lonza, Verviers, Belgium), $1 \%$ of antibiotics (10 units of penicillin, $10 \mathrm{mg}$ of streptomycin, $25 \mu \mathrm{g}$ of amphotericin B/mL; Sigma-Aldrich, Saint Louis, USA), $1 \%$ of non-essential amino acids (Lonza) and $1 \%$ of sodium pyruvate (Lonza). THP-1 macrophages were grown in Roswell Park Memorial Institute (RPMI) 1640 medium (Lonza) supplemented with adapted substrates as described in the ATCC protocol. Cell lines were cultured according to the ATCC protocol and maintained at $37^{\circ} \mathrm{C}$ in a humidified atmosphere with $5 \%$ $\mathrm{CO}_{2}$. THP-1 cell adherence and differentiation into 24-well plates was allowed by medium supplemented with $100 \mathrm{mM}$ phorbol 12 myristate 13-acetate (PMA, Sigma, Saint-Quentin Fallavier, France) for $48 \mathrm{~h}$.

\subsection{Transfection}

U87MG cells were seeded onto 24-well plates at the density of $5 \times 10^{4}$ cells/well and precultured overnight. Before transfection, the medium was changed to a fresh one containing no serum. Treatment with different LNCs (blank, liposome, scrambled and EGFR siRNA) were realized at a final dose of 37.5-300 ng siRNA/well and removed $4 \mathrm{~h}$ post-transfection. These cells were subsequently cultured for an additional time and the medium was removed every two days.

\subsection{Flow cytometry}

Immunostaining was performed to evaluate the expression of membranous EGFR protein. For this, $96 \mathrm{~h}$ after transfection, cells were seeded in 96-well plate, washed twice with PBS (lonza), and 
incubated with primary antibodies (EGFR or isotype) for $1 \mathrm{~h}$ at $4{ }^{\circ} \mathrm{C}$. After a second wash with PBS/fetal calf serum/azide $0.02 \%$, secondary antibody coupled with FITC (polyclonal goat antimouse Ig/FITC, goat F(ab2'), Dako, Trappes, France) was incubated for $30 \mathrm{~min}$ at $4{ }^{\circ} \mathrm{C}$ and washed. Cell suspensions were fixed with $\mathrm{PBS} /$ azide $0.02 \% /$ formaldehyde $1 \%$, protected from light and conserved at $4{ }^{\circ} \mathrm{C}$. Analyses were performed with a FACScalibur flow cytometer (BD Bioscience, San José, USA) in collaboration with SCCAN platform (Angers).

\subsection{Survival assay}

Glioma cells and macrophages were seeded onto 24-well plates at a density of $5 \times 10^{4}$ cells/well and pre-cultured overnight or $48 \mathrm{~h}$ for THP-1. Dose-dependent transfection was realized as described above to determine the $\mathrm{IC}_{50}$ and cells were subsequently cultured for two days. Cytotoxicity assays were performed using MTS (3-(4,5-dimethylthiazol-2-yl)-5-(3-carboxymethoxyphenyl)2-(4-sulfophenyl)-2Htetrazolium) (Promega, Madison, USA). For this, $100 \mu \mathrm{L}$ of MTS/well were disposed and plates were incubated $2.5-4 \mathrm{~h}$ at $37^{\circ} \mathrm{C}$ in a humidified atmosphere with $5 \% \mathrm{CO}_{2}$. The OD at $492 \mathrm{~nm}$ was evaluated by Mutliskan Ascent (Labsystems, Fisher Scientific, Wilmington, USA).

\subsection{Proliferation study}

Glioma cells were seeded onto 24-well plates at a density of $5 \times 10^{4}$ cells/well and pre-cultured overnight. A time-dependent transfection with the low dose (10 ng) was realized and cells were subsequently cultured for ten days and the medium was removed every two days. To evaluate the progression of the glioma cells, sphere formation was followed every day. For this, scores were defined: 0 corresponding to the absence of a sphere in a well, 1: irregular apparition of small spheres, 2: regular distribution of small spheres, 3: regular medium spheres, 4: regular large spheres. The cells were photographed at day 10 after treatment. Moreover, every two day, cells were suspended by trypsin $(0.5 \mathrm{~g}$ porcin trypsin, 0.2 g EDTA; Sigma, Saint Louis, USA), and two washes were realized with PBS. Analyses with trypan blue staining was used to count the number of cells per well.

\subsection{Complement test}

Complement consumption was assessed in normal human serum (NHS) (provided by the Etablissement Francais du Sang, $\mathrm{CHU}$, Angers, France) by measuring the residual hemolytic capacity of the complement system after contact with LNCs. The final dilution of NHS in the mixture was $1: 4(\mathrm{~V} / \mathrm{V})$ corresponding to $100 \mu \mathrm{L}$ of NHS into $400 \mu \mathrm{L}$ of reactive media. The technique consisted in determining the amount of serum able to lyse $50 \%$ of a fixed number of sensitized sheep erythrocytes with rabbit antisheep erythrocyte antibodies ( $\mathrm{CH} 50$ ), according to the procedure described by Vonarbourg et al. (2009). Complement activation was first expressed as a function of the surface area in order to compare different formulations. Nanoparticle surface areas were estimated using the equation: $S=3 \mathrm{~m} / \mathrm{r} \rho$, where $S$ is the surface area $\left[\mathrm{cm}^{2}\right], m$ is the weight [g] in $1 \mathrm{~mL}$ of suspension, $r$ is the average radius [ $\mathrm{cm}$ ] determined by DLS, and $\rho$ is the volumetric mass $\left[\mathrm{g} / \mathrm{cm}^{3}\right]$ of the nanoparticles estimated at $100 \mathrm{~g} / \mathrm{cm}^{3}$.

\subsection{Statistical analyses}

Comparisons between the treated cells of all groups were performed either using a classical analysis of variance (one-way
ANOVA) followed by a Scheffe's post hoc analysis. Statistical significance was ascribed to a threshold $p$-value of 0.05 .

\section{Results}

\subsection{SiRNA LNC formulation}

\subsubsection{Size and Zeta potential}

The size and Zeta potential of siRNA LNCs were determined using a Nanosizer apparatus. Measurements were realized 1 day after formulation and 1 month after storage at $4{ }^{\circ} \mathrm{C}$ to evaluate the physico-chemical stability of siRNA LNCs (Table 1). Blank LNCs exhibited a stable size, narrow distribution around $60 \mathrm{~nm}$ and a negative Zeta potential estimated at $-5 \mathrm{mV}$ over a 1 month period. Liposome LNCs showed a decreasing evolution in size and an increasing surface charge after 1 month at $4{ }^{\circ} \mathrm{C}$. The Zeta potential was largely positive due to cationic lipids and their polydispersity index (PdI) that decreased strongly with time from 0.26 to 0.05 . Concerning the two different lipoplexe CR used to obtain siRNA LNCs, an influence was observed with an increase of size after formulation with $90 \mathrm{~nm}$ and $122 \mathrm{~nm}$ respectively for CR of 2.5 and 5 compared to blank LNCs $(56 \mathrm{~nm})$. Moreover, this parameter was associated to a high PdI just inferior to 0.3 (limit of monodispersity). As observed with time for liposome LNCs, siRNA LNCs presented a high reduction of size and PdI with a gain of Zeta potential (Table 1).

\subsubsection{Encapsulation efficiency}

An adapted method based on UV spectroscopy was used to evaluate the encapsulation efficiency of siRNA into LNCs. Briefly; four compartments of siRNA localization (free siRNA, free lipoplexes, encapsulated siRNA and encapsulated lipoplexes) were separated using an easy chloroform extraction as described above. Analyses of EGFR siRNA LNC encapsulation efficiency were performed, in a similar way as for size, one day and 1 month after formulation (Table 1). The encapsulation was estimated at $29 \%$ and $40 \%$ for respectively CR 2.5 and 5. The stability of encapsulated doses was confirmed for both CR 1 month after formulation. The majority of encapsulated siRNA was associated with liposomes. However, a non-negligible part of siRNA was not associated with liposome but was still encapsulated into LNCs as previously observed. According to their size, Zeta potential and encapsulation stability, one day-old EGFR siRNA LNCs were used in the following experiments.

\subsection{Analysis of efficient transfection in vitro}

Four days after EGFR siRNA LNC treatment, the EGFR protein level was evaluated by flow cytometry (Fig. 1). No protein extinction was observed with siRNA alone in opposition to what was observed with siRNA associated with a commercial transfection agent (ICAfectin ${ }^{\circledR}$, Invitrogen, Saint Aubin, France) which resulted in a 55\% of inhibition with a low dose of siRNA (37.5 ng) (Fig. 1). Liposome and scrambled LNCs showed no difference of EGFR expression compared to non-treated cells. Moreover, CR 2.5 LNCs had no effect on U87MG EGFR expression until $300 \mathrm{ng}$, the maximal dose (Fig. 1A). CR 5 LNCs demonstrated a dose-dependent response with a maximal inhibition estimated at $63 \%$, equivalent to the positive control result (Fig. 1B). Consequently, CR 5 LNCs were chosen to perform in vitro assays.

\subsection{Determination of $I C_{50}$}

The cytotoxicity of EGFR siRNA LNCs was performed on two cell lines: U87MG glioma and THP-1 human cells in differentiated macrophages. EGFR siRNA had only a little impact on U87MG cell 
Table 1

Characterization of EGFR siRNA LNCs.

\begin{tabular}{|c|c|c|c|c|c|c|}
\hline Type of LNCs & Liposome type and CR & Date & Size $(n m)$ & PdI & Zeta $(\mathrm{mV})$ & Encapsulation yield (\%) \\
\hline \multirow{4}{*}{ EGFR siRNA LNCs } & \multirow[t]{2}{*}{ DOTAP/DOPE 2.5} & 1 day & $90 \pm 1$ & $0.22 \pm 0.01$ & $+1 \pm 1$ & $29 \pm 9$ \\
\hline & & 1 month & $56 \pm 1$ & $0.09 \pm 0.02$ & $+24 \pm 5$ & $24 \pm 3$ \\
\hline & \multirow{2}{*}{ DOTAP/DOPE 5} & 1 day & $122 \pm 1$ & $0.26 \pm 0.01$ & $+4 \pm 1$ & $40 \pm 12$ \\
\hline & & 1 month & $70 \pm 1$ & $0.06 \pm 0.01$ & $+26 \pm 1$ & $42 \pm 7$ \\
\hline \multirow{2}{*}{ Liposome LNCs } & \multirow[t]{2}{*}{ DOTAP/DOPE 5} & 1 day & $102 \pm 10$ & $0.26 \pm 0.01$ & $+5 \pm 2$ & 1 \\
\hline & & 1 month & $58 \pm 2$ & $0.05 \pm 0.01$ & $+34 \pm 2$ & 1 \\
\hline \multirow{2}{*}{ Blank LNCs } & \multirow[t]{2}{*}{ I } & 1 day & $56 \pm 1$ & $0.04 \pm 0.01$ & $-6 \pm 1$ & l \\
\hline & & 1 month & $60 \pm 2$ & $0.03 \pm 0.01$ & $-5 \pm 1$ & 1 \\
\hline
\end{tabular}

CR: charge ratio, PdI: polydispersity index (monodispersity: $\mathrm{PdI}<0.3$ ).

viability, with a low $\mathrm{IC}_{50}$ evaluated at $40 \mathrm{ng}$ (Fig. 2). This effect was considered specific since the survival of differentiated THP1 cells was not affected by the EGFR siRNA. Toxicity of liposome and scrambled LNCs was observed at 250 and $80 \mathrm{ng}$ on U87MG (Fig. 2A) and 70 and 120 ng on THP-1 cells respectively (Fig. 2B). Following these results, a dose of $10 \mathrm{ng}$ siRNA corresponding to $0.1 \mathrm{mg} / \mathrm{mL}$ of LNCs was chosen to study the growth progression for 10 days as it led to cytotoxicity (30\%) compared to control conditions.
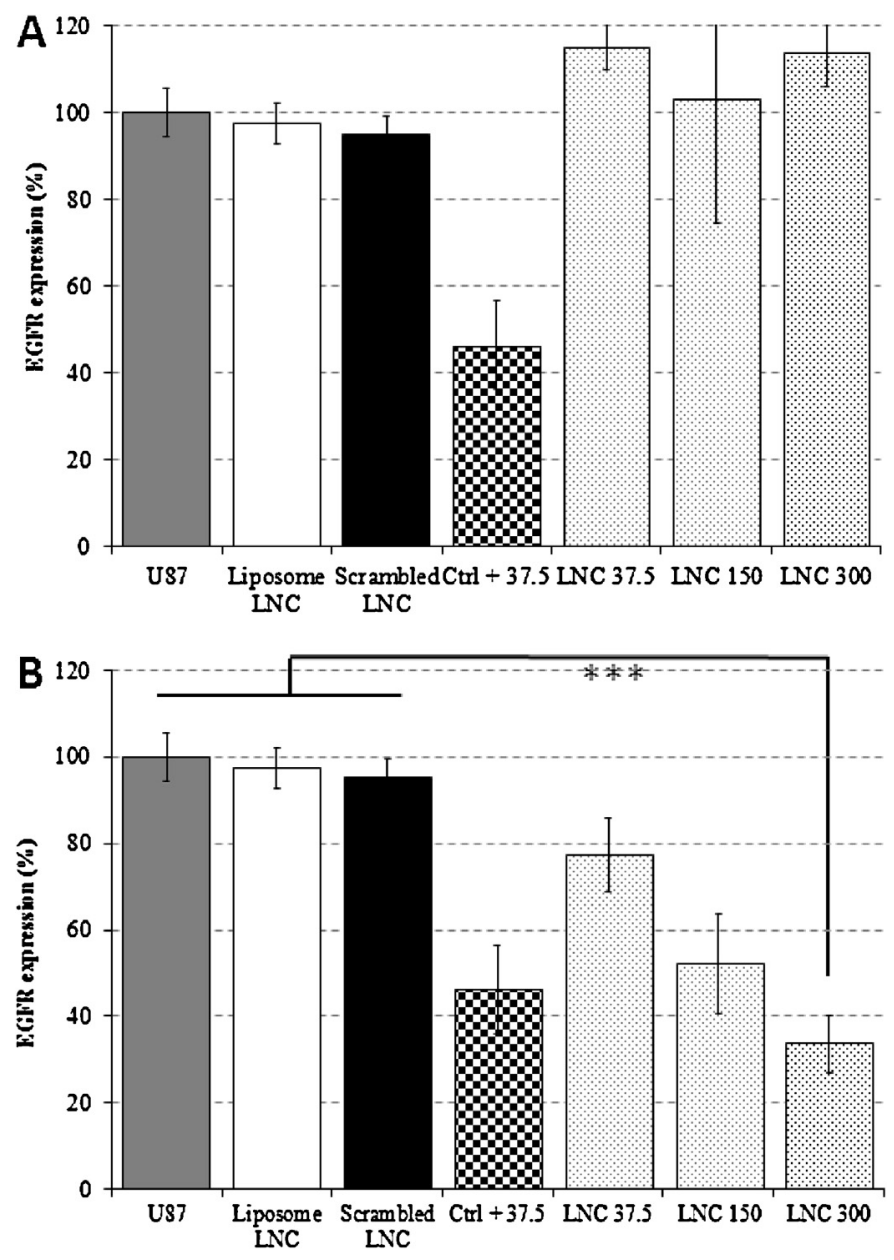

Fig. 1. Expression of EGFR protein by flow cytometry analyses after treatment with two CR of EGFR siRNA LNCs. These analyses by flow cytometry of EGFR levels were realized four days after treatment with increasing doses comprised between $37.5 \mathrm{ng}$ and $300 \mathrm{ng}$ of siRNA per well. The EGFR protein level was expressed in function of non-treated cells. (A) Analyses of DOTAP/DOPE CR 2.5 LNCs. (B) Analyses of DOTAP/DOPE CR 5 LNCs. Statistical analyses ANOVA one way post hoc Scheffe, ${ }^{* * *} p=0.001$.

\subsection{Proliferation impact}

After 10 days, pictures of U87MG cells were taken (Fig. 3A). The apparition of spheres into wells was recorded as described above in the cell proliferation study. A different organization of U87MG cells was evidenced between non-treated cells (a), cells treated with liposome LNCs (b) and scrambled LNCs (c) compared to cells that received EGFR siRNA LNCs (d) (Fig. 3A). EGFR siRNA LNCs significantly decreased the formation of U87MG spheres from Days 6 to 10 (Fig. 3B). Liposome and scrambled LNCs followed the same
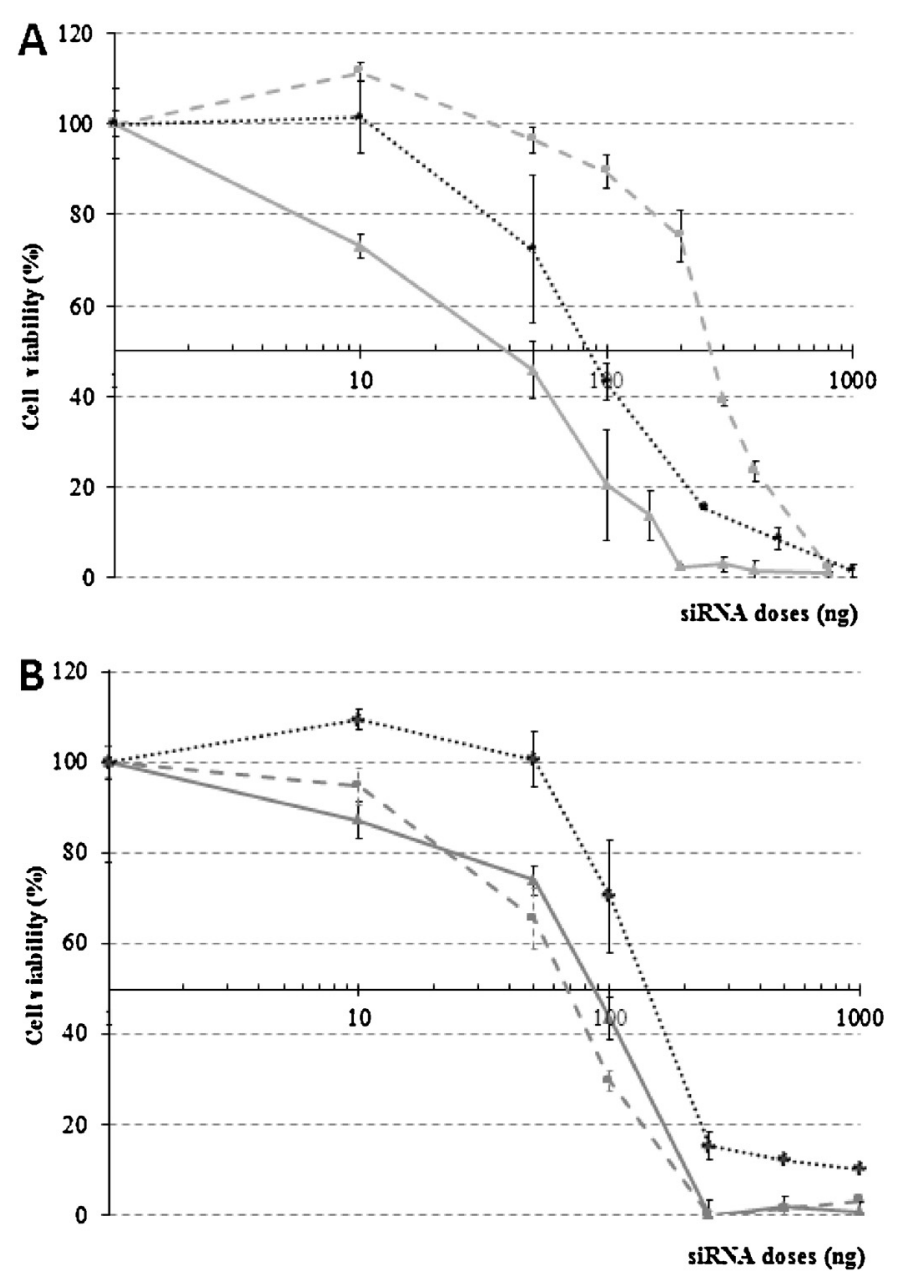

Fig. 2. Determination of $I_{50}$ values of EGFR siRNA LNCs on human glioma cells (U87MG) (A) and differentiated macrophages (THP-1) (B). (A) EGFR siRNA into LNCs ( ) had an impact on U87MG viability with $\mathrm{IC}_{50}$ estimated lower than $40 \mathrm{ng}$ of siRNA compared to control conditions with IC50 superior at $80 \mathrm{ng}$ (liposome $-{ }^{-}-$and scrambled siRNA LNCs $\cdot \cdot-\cdot \cdot$ ). (B) On macrophages, EGFR siRNA LNCs did not modify the cell viability. 

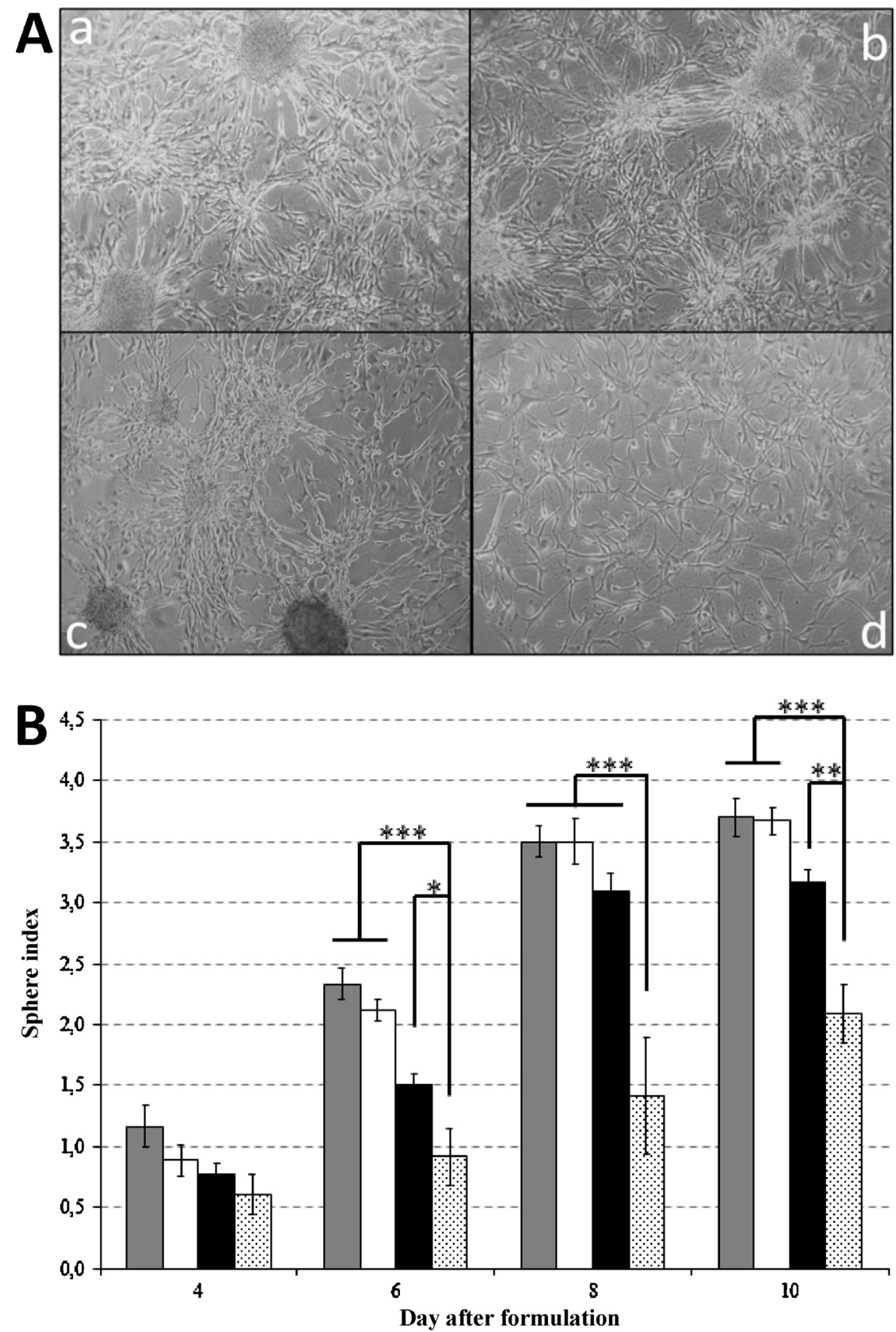

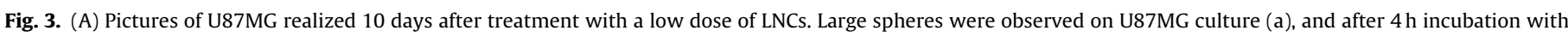

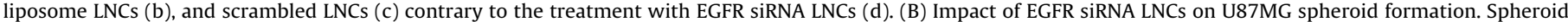

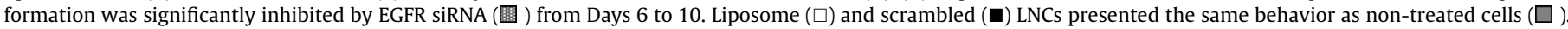
Statistical analyses ANOVA one way post hoc Scheffe, ${ }^{*} p=0.05,{ }^{* *} p=0.01$ and ${ }^{* * *} p=0.001$.

progression as non-treated cells with maximal index obtained at day 10 (Fig. 3B). To confirm this result, a determination of cell numbers was realized in parallel. EGFR LNC treatment presented a significant difference with all controls at day 10 (Fig. 4). On the final day, a difference above $30 \%$ was evidenced between EGFR siRNA LNCs and all control LNCs.

\subsection{Complement activation}

The diameter of nanoparticles was controlled by a classical method before performing $\mathrm{CH} 50$ assays (Fig. 5). The analysis of complement proteins activation was performed on liposomes, lipoplexes, blank LNCs, liposome LNCs, and EGFR siRNA LNCs to compare their behavior in contact with human serum (Fig. 5). No activation of complement proteins was evidenced with siRNA alone (data not shown). The very low activation profile of blank LNCs was already described due to negative surface charge and a size of around $60 \mathrm{~nm}$ (Morille et al., 2010). In opposition to LNCs, positively-charged surface delivery system such as liposome and lipoplexes led to a high consumption of complement proteins with higher profile for lipoplexes. Thanks to the neutral charge of our device, intermediate consumption was evidenced for loaded LNCs 


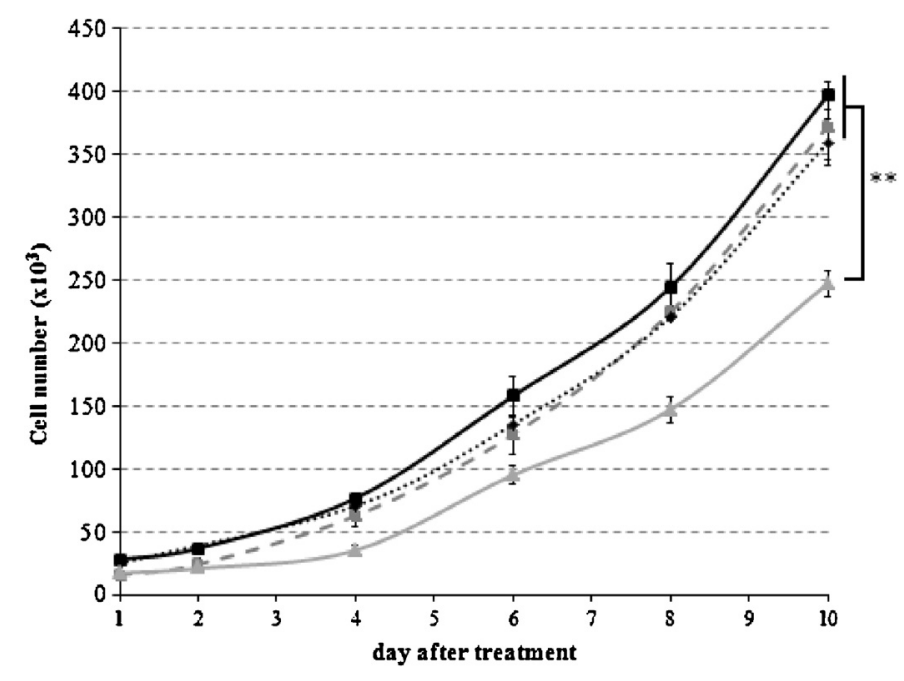

Fig. 4. Evolution of U87MG cellular proliferation after one treatment with $10 \mathrm{ng}$ of EGFR siRNA LNCs. This dose allowed a decrease of cell numbers with EGFR siRNA LNCs $(--$ ) compared to other treatments such as liposome LNCs $(-\square-$ ) and scrambled siRNA LNCs (..... ) from Day 5 to Day 10. A significant difference with non-treated cells (—— ) was evidenced on Day 10. Statistical analyses ANOVA one way post hoc Scheffe, ${ }^{* *} p=0.01$.

(liposome and siRNA), which were close to the blank LNC profile until $400-500 \mathrm{~cm}^{2}$.

\section{Discussion}

In this study, we developed a specific delivery system targeting EGFR protein by using an RNAi mechanism for glioma therapy. This nanomedicine consists of the association of DOTAP/DOPE siRNA cationic lipoplexes and lipid nanocapsules (LNCs) to combine (i) the efficient electrostatic interaction of lipids and siRNA, (ii) the high transfection capacity of cationic lipids and (iii) the systemic delivery of nanocapsules thanks to their stealth property (Vonarbourg et al., 2009). DOTAP and DOPE lipids have been commonly used as transfection agents and as components in nucleic acid delivery systems (Resnier et al., 2013). In this work, two different charge ratios (2.5 and 5) were studied to follow their impact on siRNA LNC formulation. These two formulations presented a size inferior to $150 \mathrm{~nm}$, a neutral Zeta potential and an encapsulation stability adequate for a long circulating profile. However, measures realized 1 month later highlighted the change of physicochemical properties of siRNA LNCs over time. Size diminution and surface charge increases could signify that LNCs were reorganized in positive smaller particles, possibly due to the large amount of cationic tensio-active molecules (Lecithin, DOTAP and DOPE). This point could also explain the difference of size between siRNA LNCs CR 2.5 and 5. Twice as many lipids were introduced into the CR 5 formulation compared to $C R$ 2.5 , which could lead to larger nanoparticles. Despite this phenomenon, the encapsulated part of siRNA was unchanged and the high CR was more interesting because it led to higher encapsulation efficiency, estimated at $40 \%$ of initial quantity of siRNA, which corresponds to a concentration of $100 \mu \mathrm{g}$ of encapsulated siRNA per $\mathrm{mL}$ of LNCs (=300 $\mathrm{mg}$ of LNCs).

The protein inhibition study confirmed the benefit of using this high charge ratio during our formulation. Indeed, it resulted in an extinction of membranous EGFR evaluated at 63\%. This high extinction percentage was most likely due to our nanomedicine composition. Indeed, lipid components of siRNA LNCs, i.e., cationic lipids and lecithin, could promote the passage through cell membranes by their hydrophilic polar heads. The slightly positive surface charge of siRNA LNCs is expected to favor the electrostatic interactions between nanocapsules and cell membrane to facilitate their internalization. In fact, LNCs are known to be well-internalized by cancer cells, especially for the larger ones $(100 \mathrm{~nm})$ by multiple, non-receptor-dependent internalization pathways such as clathrin, endocytosis and cavoelae pathways (Paillard et al., 2010). Finally, a major role of these lipids in endosomal escape has already been demonstrated, especially for DOTAP and DOPE lipids involved in two well-known mechanisms: the proton sponge effect and membrane destabilization (Cardarelli et al., 2012; Varkouhi et al., 2011).

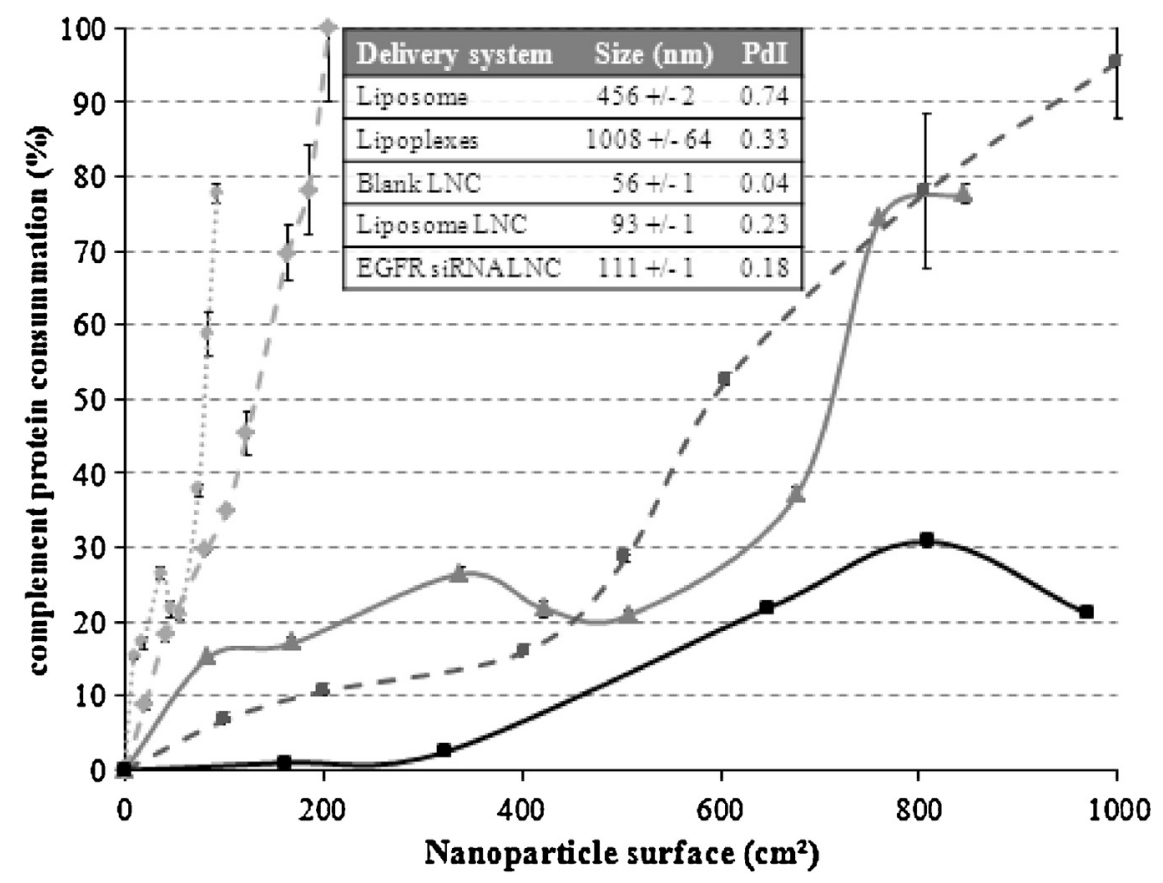

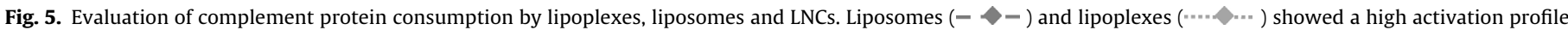

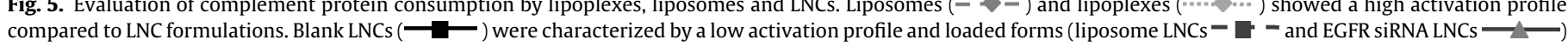
presented an intermediate profile. 
Combined with the initial properties of LNCs concerning their endosomal escape capacities, these lipids could favor this phenomenon (Paillard et al., 2010).

In the literature, the inhibition of EGFR in glioma models was assessed by shRNA form, another RNAi strategy. ShRNAs correspond to an intermediate form before mature siRNA is formed, and are characterized by a hairpin loop structure. However, shRNAs are not stable, so their use is generally replaced by a DNA plasmid encoding for these shRNAs. The siRNA LNCs showed a similar extinction of EGFR protein as the shRNA EGFR used in combination with classic transfection agents (Inaba et al., 2011). PAMAM dendrimers, used for shRNA plasmid transfection, showed after $48 \mathrm{~h}$ transfection an inhibition of EGFR lower than siRNA LNCs on glioma cells (35\% versus 63\%) (Han et al., 2010). Moreover, cell proliferation was significantly reduced by our siRNA EGFR LNCs after only 10 days of treatment. Electroporation of shRNA plasmid resulted to similar results with a $40 \%$ of cell density decrease after 6 days. However this transfection methodology, i.e. electroporation, is not translatable to clinical situations.

The benefit of LNCs is their possible use for intravenous injection as we validated their capacity to protect siRNA from complement opsonisation by $\mathrm{CH} 50$ assay. In fact, the low activation profile obtained in complement activation experiments confirmed the possible capacity of siRNA LNCs to circulate a long time in blood. This result can be explained by the benefit of the nanocapsule shell that hides the positive charges of the lipids thanks to weak links with negative PEG dipoles (Vonarbourg et al., 2005), thereby conferring stealth property to our LNCs in opposition to lipoplexes. This great enhancement in the behavior of our LNCs is more likely due to the presence of PEG chains at the surface of LNCs, as demonstrated in our previous works on DNA LNCs (David et al., 2012a). To evaluate this, in vivo experiments should be realized to define the half-life time and biodistribution of siRNA LNCs in a murine glioma model.

Interestingly, EGFR expression has also been described to be involved in chemo and radioresistance phenomena (Watanabe et al., 1996). For this, the potential of combinatory treatments could be assessed like Chen and co-workers did with doxorubicin and cmyc siRNA (Chen et al., 2010). In the future, the impact of siRNA EGFR inhibition on sensitization of classic chemotherapeutic agent or radiation could be studied in vitro and in vivo.

\section{Conclusion}

Our lipid nanocapsules (LNCs) were created 10 years ago by an easy process formulation and have now been fully characterized. Moreover, previous studies have demonstrated the possibility to encapsulate hydrophilic nucleic acids into these systems. Here, our first aim consisted of the study of the impact of CR used and the stability depending on EGFR siRNA LNC physico-chemical parameters. Analyses of protein levels allowed us to conclude on the promising delivery of siRNA into the cytoplasm by LNCs thanks to the high degree of inhibition observed. The potential of EGFR siRNA targeting to combat glioma progression was evidenced on cell growth and U87MG sphere development. Finally, stealth properties evaluated by $\mathrm{CH} 50$ assay, confirmed for EGFR siRNA LNCs, the possibility of future treatment to combat glioma cell proliferation alone or as an adjuvant treatment by systemic injection.

\section{Acknowledgments}

This work was supported by the foundation "Association de Recherche contre le Cancer" and by "La Ligue contre le cancer 49 et 35". We would like to thank the SFR "Structure Fédérative de Recherche - Interactions cellulaires et applications thérapeutiques" and the active participation of SCCAN platform, especially Dr Catherine Guillet for flow cytometry analyses.

\section{References}

Beduneau, A., Hindre, F., Clavreul, A., Leroux, J.C., Saulnier, P., Benoit, J.P., 2008. Brain targeting using novel lipid nanovectors. J. Control. Release 126, 44-49.

Cardarelli, F., Pozzi, D., Bifone, A., Marchini, C., Caracciolo, G., 2012. Cholesteroldependent macropinocytosis and endosomal escape control the transfection efficiency of lipoplexes in CHO living cells. Mol. Pharm. 9, 334-340.

Chen, Y., Wu, J.J., Huang, L., 2010. Nanoparticles targeted with NGR motif deliver c-myc siRNA and doxorubicin for anticancer therapy. Mol. Ther. 18, 828-834.

David, S., Carmoy, N., Resnier, P., Denis, C., Misery, L., Pitard, B., Benoit, J.P., Passirani, C., Montier, T., 2012a. In vivo imaging of DNA lipid nanocapsules after systemic administration in a melanoma mouse model. Int. J. Pharm. 423, 108-115.

David, S., Montier, T., Carmoy, N., Resnier, P., Clavreul, A., Mevel, M., Pitard, B., Benoit, J.P., Passirani, C., 2012b. Treatment efficacy of DNA lipid nanocapsules and DNA multimodular systems after systemic administration in a human glioma model. J. Gene Med. 14, 769-775.

David, S., Resnier, P., Guillot, A., Pitard, B., Benoit, J.P., Passirani, C., 2012c. siRNA LNCs - a novel platform of lipid nanocapsules for systemic siRNA administration. Eur. J. Pharm. Biopharm. 81, 448-452.

Fire, A., Xu, S., Montgomery, M.K., Kostas, S.A., Driver, S.E., Mello, C.C., 1998. Potent and specific genetic interference by double-stranded RNA in Caenorhabditis elegans. Nature 391, 806-811.

Geoerger, B., Gaspar, N., Opolon, P., Morizet, J., Devanz, P., Lecluse, Y., Valent, A., Lacroix, L., Grill, J., Vassal, G., 2008. EGFR tyrosine kinase inhibition radiosensitizes and induces apoptosis in malignant glioma and childhood ependymoma xenografts. Int. J. Cancer 123, 209-216.

Han, L., Zhang, A., Wang, H., Pu, P., Jiang, X., Kang, C., Chang, J., 2010. Tat-BMPsPAMAM conjugates enhance therapeutic effect of small interference RNA on U251 glioma cells in vitro and in vivo. Hum. Gene Ther. 21, 417-426.

Hatanpaa, K.J., Burma, S., Zhao, D., Habib, A.A., 2010. Epidermal growth factor receptor in glioma: signal transduction, neuropathology, imaging, and radioresistance. Neoplasia 12,675-684.

Heurtault, B., Saulnier, P., Pech, B., Proust, J.E., Benoit, J.P., 2002. A novel phase inversion-based process for the preparation of lipid nanocarriers. Pharm. Res. $19,875-880$.

Inaba, N., Fujioka, K., Saito, H., Kimura, M., Ikeda, K., Inoue, Y., Ishizawa, S., Manome, Y., 2011. Down-regulation of EGFR prolonged cell growth of glioma but did not increase the sensitivity to temozolomide. Anticancer Res. 31, 3253-3257.

Jin, X., Jin, X., Sohn, Y.W., Yin, J., Kim, S.H., Joshi, K., Nam, D.H., Nakano, I., Kim, H., 2013. Blockade of EGFR signaling promotes glioma stem-like cell invasiveness by abolishing ID3-mediated inhibition of p27(KIP1) and MMP3 expression. Cancer Lett. 328, 235-242.

Joshi, A.D., Loilome, W., Siu, I.M., Tyler, B., Gallia, G.L., Riggins, G.J., 2012. Evaluation of tyrosine kinase inhibitor combinations for glioblastoma therapy. PLoS ONE 7, e44372.

Maeda, H., 2001. The enhanced permeability and retention (EPR) effect in tumor vasculature: the key role of tumor-selective macromolecular drug targeting. Adv. Enzyme Regul. 41, 189-207.

Michiue, H., Eguchi, A., Scadeng, M., Dowdy, S.F., 2009. Induction of in vivo synthetic lethal RNAi responses to treat glioblastoma. Cancer Biol. Ther. 8, 2306-2313.

Morille, M., Montier, T., Legras, P., Carmoy, N., Brodin, P., Pitard, B., Benoit, J.P., Passirani, C., 2010. Long-circulating DNA lipid nanocapsules as new vector for passive tumor targeting. Biomaterials 31, 321-329.

Morille, M., Passirani, C., Dufort, S., Bastiat, G., Pitard, B., Coll, J.L., Benoit, J.P., 2011. Tumor transfection after systemic injection of DNA lipid nanocapsules. Biomaterials 32, 2327-2333.

Paillard, A., Hindre, F., Vignes-Colombeix, C., Benoit, J.P., Garcion, E., 2010. The importance of endo-lysosomal escape with lipid nanocapsules for drug subcellular bioavailability. Biomaterials 31, 7542-7554.

Resnier, P., Montier, T., Mathieu, V., Benoit, J.-P., Passirani, C., 2013. Current status of siRNA delivery systems to combat cancers: from needs and challenges to opportunities and clinical perspectives. Adv. Drug Deliv. Rev..

Stupp, R., Roila, F., 2009. Malignant glioma: ESMO clinical recommendations for diagnosis, treatment and follow-up. Ann. Oncol. 20, 126-128.

Taylor, T.E., Furnari, F.B., Cavenee, W.K., 2012. Targeting EGFR for treatment of glioblastoma: molecular basis to overcome resistance. Curr. Cancer Drug Targets 12, 197-209.

Thiessen, B., Stewart, C., Tsao, M., Kamel-Reid, S., Schaiquevich, P., Mason, W., Easaw, J., Belanger, K., Forsyth, P., McIntosh, L., Eisenhauer, E., 2010. A phase I/II trial of GW572016 (lapatinib) in recurrent glioblastoma multiforme: clinical outcomes, pharmacokinetics and molecular correlation. Cancer Chemother. Pharmacol. 65, 353-361.

Varkouhi, A.K., Scholte, M., Storm, G., Haisma, H.J., 2011. Endosomal escape pathways for delivery of biologicals. J. Control. Release 151, 220-228.

Vonarbourg, A., Passirani, C., Desigaux, L., Allard, E., Saulnier, P., Lambert, O., Benoit, J.P., Pitard, B., 2009. The encapsulation of DNA molecules within biomimetic lipid nanocapsules. Biomaterials 30, 3197-3204. 
Vonarbourg, A., Saulnier, P., Passirani, C., Benoit, J.P., 2005. Electrokinetic properties of noncharged lipid nanocapsules: influence of the dipolar distribution at the interface. Electrophoresis 26, 2066-2075.

Wang, T., Agarwal, S., Elmquist, W.F., 2012. Brain distribution of cediranib is limited by active efflux at the blood-brain barrier. J. Pharmacol. Exp. Ther. 341, 386-395.

Watanabe, K., Tachibana, O., Sata, K., Yonekawa, Y., Kleihues, P., Ohgaki, H., 1996. Overexpression of the EGF receptor and p53 mutations are mutually exclusive in the evolution of primary and secondary glioblastomas. Brain. Pathol. 6, 21-223, discussion 23-24.

Yang, W., Xia, Y., Cao, Y., Zheng, Y., Bu, W., Zhang, L., You, M.J., Koh, M.Y., Cote, G., Aldape, K., Li, Y., Verma, I.M., Chiao, P.J., Lu, Z., 2012. EGFR-induced and PKCepsilon monoubiquitylation-dependent NF-kappaB activation upregulates PKM2 expression and promotes tumorigenesis. Mol. Cell 48, 771-784. 